\title{
Meta-analysis and indirect treatment comparison of modified FOLFIRINOX and gemcitabine plus nab- paclitaxel as first-line chemotherapy in advanced pancreatic cancer
}

\section{Jiayuan Chen}

Cancer center, Union Hospital, Huazhong University of Science and Technology

Qingling Hua

Cancer center, Union Hospital, Huazhong University and Technology

\section{Haihong Wang}

Cancer center, Union Hospital, Huazhong University of Science and Technology

\section{Dejun Zhang}

Cancer center, Union Hospital, Huazhong University of Science and Technology

\section{Lei Zhao}

Cancer center, Union Hospital, Huazhong University of Science and Technology

\section{Dandan $\mathrm{Yu}$}

Cancer center, Union Hospital, Huazhong University of Science and Technology

\section{Guoliang Pi}

Department of Radiation Oncology, Hubei Cancer Hospital, Tongji Medical College, Huazhong University of Science and Technology

\section{Tao Zhang ( $\square$ xhzlzt@126.com )}

Cancer center, Union Hospital, Huazhong University of Science and Technology

\section{Zhenyu Lin}

Cancer center, Union Hospital, Huazhong University of Science and Technology

\section{Research article}

Keywords: pancreatic cancer, modified FOLFIRINOX, gemcitabine, nab-paclitaxel, network meta-analysis, systematic review

Posted Date: June 22nd, 2020

DOI: https://doi.org/10.21203/rs.3.rs-37084/v1

License: (c) (1) This work is licensed under a Creative Commons Attribution 4.0 International License. Read Full License 
Version of Record: A version of this preprint was published at BMC Cancer on July 23rd, 2021. See the published version at https://doi.org/10.1186/s12885-021-08605-x. 


\section{Abstract}

Background: Modified FOLFIRINOX and gemcitabine plus nab-paclitaxel (GEM-NAB) have been recommended as first-line therapies for advanced pancreatic cancer (PC) at present. Due to the lack of direct comparison, we conducted this network meta-analysis to indirectly compare the effectiveness and toxicity of modified FOLFIRINOX and GEM-NAB.

Methods: The eligible retrospective studies of treatments related to modified FOLFIRINOX and GEM-NAB were searched up to 4 April 2020. We used the frequentist model to analyze the survival and toxicity data between different treatments. Pooled analysis for overall survival (OS), progression-free survival (PFS), objective response rate (ORR) and events of toxicity were analyzed in this study.

Results: Twenty-two studies including 6351 patients were involved in this network meta-analysis. The comparisons based on OS and PFS showed that modified FOLFIRINOX and GEM-NAB had similar treatment efficacy. But GEM-NAB was more effective than modified FOLFIRINOX based on the result of ORR (RR: 0.70; 95\% Cl: 0.51-0.96). Moreover, our analysis showed a similar toxicity profile between modified FOLFIRINOX and GEM-NAB.

Conclusions: Based on current evidence, modified FOLFIRINOX and GEM-NAB were similar in survival and toxicity comparisons. Many factors should be considered for the optimal treatment formulation and our meta-analysis could provide some guidance to treatment selection in the first-line setting for advanced PC.

\section{Background}

Among cancer-related deaths, pancreatic cancer (PC) ranked fourth in both men and women in the United States. Because most patients were diagnosed at advanced stage, the prognosis was poor with a 5-year survival rate less than $7 \%$ despite significant improvements in therapeutic strategies. There will be an estimated 57,600 new cases and 47,050 deaths with PC in 2020[1]. As curative surgery could only be performed in less than $20 \%$ of cases, chemotherapy was commonly used in patients with locally advanced or metastatic PC. Gemcitabine (GEM) were found to have more clinical benefits than 5fluorouracil (5-FU) for treating advanced PC in a clinical trial and became one of the main chemotherapeutic drugs. And chemotherapies based on gemcitabine or fluoropyrimidine are conventional treatments for advanced PC[2,3]. Different anti-tumor agents were combined with gemcitabine or fluoropyrimidine in numerous clinical trials to improve treatment efficacy. And gemcitabine combined with nab-paclitaxel prolonged OS for around two months than gemcitabine monotherapy[4, 5].

Among all regimen combinations, FOLFIRINOX (folinic acid, 5-FU, irinotecan and oxaliplatin) and GEMNAB (gemcitabine plus nab-paclitaxel) have shown great benefits as first-line therapies for advanced PC at present. In general, GEM-NAB is more tolerable and preferred by older patients with a higher ECOG PS, while FOLFIRINOX is often used in younger patients[6, 7]. Due to drug toxicities, dosage modifications have been made in FOLFIRINOX and modified FOLFIRINOX have been recommended in several 
institutions[8, 9]. In particular, evidence was lacked directly assessing benefits or adverse effects in patients who received modified FOLFIRINOX or GEM-NAB as first-line treatments. Thus, we collected data available from observational retrospective studies and performed a systematic review and network metaanalysis in this article. We aimed to compare the effectiveness and toxicity of modified FOLFIRINOX and GEM-NAB indirectly in the first-line setting.

\section{Methods}

\section{Literature search and article selection}

We conducted a systematic search to find available papers in literature. The databases including PubMed, Embase, Cochrane and Web of Science were independently searched by two investigators from inception to April 2020. We utilized the following keywords for this search: pancreatic cancer, gemcitabine, nab-paclitaxel, and modified FOLFIRINOX. The search strategy was as follows: (('folinic acid'/exp AND fluorouracil/exp AND irinotecan/exp AND oxaliplatin/exp AND 'drug combination'/exp) OR (Folfirinox):ab,ti) OR (gemcitabine/exp AND 'Albumin-Bound Paclitaxel'/exp 'drug combination'/exp) ) AND ('pancreas cancer'/de OR 'pancreas tumor'/de OR 'pancreas adenoma'/de OR 'pancreas adenocarcinoma'/de OR 'pancreas carcinoma'/de OR 'pancreas islet cell carcinoma'/de OR (pancrea* NEAR/3 (cancer* OR neoplas* OR tumo* OR adenocarcinom* OR carcinom* OR adenom*)):ab,ti). The Additional file 2 listed detailed search strategy.

Our search was supplemented by a manual search for relevant studies. The detailed flow diagram of inclusion and exclusion process was presented in Fig. 1. We included studies on human species written in English. We selected retrospective studies to compare the benefits and adverse effects/safety of modified FOLFIRINOX versus GEM-NAB of patients with locally advanced or metastatic PC. Standard regimen of FOLFIRINOX included oxaliplatin $85 \mathrm{mg} / \mathrm{m}^{2}$, leucovorin $400 \mathrm{mg} / \mathrm{m}^{2}$, irinotecan $180 \mathrm{mg} / \mathrm{m}^{2}, 5$-FU bolus $400 \mathrm{mg} / \mathrm{m}^{2}$ and $5-\mathrm{FU} 2400 \mathrm{mg} / \mathrm{m}^{2}$. The dose of one regimen at least was reduced in modified FOLFIRINOX with/without the removal of 5-FU bolus[10]. We excluded studies if they were duplicates, reviews, case reports, meta-analysis or in no association with our research.

\section{Data Extraction}

General information was extracted from the selected studies by two investigators. Any disagreements regarding the extraction of data were resolved by discussions among several investigators. The following fields were abstracted: the name of article, the name of first author, country, year of publication, number of patients, characteristics of patients (age and sex), beginning and ending time, median followed-up time, treatment efficacy data as well as safety data.

Overall survival (OS) was the primary endpoint. Progression-free survival (PFS), objective response rate (ORR), and grade $\geq 3$ adverse events (according to National Cancer Institute Common Terminology Criteria for Adverse Events version 4.0) were the secondary endpoints. The hazard ratios (HRs) with 95\% confidence intervals (Cls) of PFS and OS were extracted from the selected publications. The total events 
of complete response (CR), partial response (PR), stable disease (SD), and progressive disease (PD) were also extracted to evaluate ORR to chemotherapy. The grade $\geq 3$ adverse events included neutropenia, febrile neutropenia, thrombocytopenia, anemia, anorexia, fatigue, nausea, and diarrhea.

\section{Statistical analysis}

Frequentist model was used in this analysis[11, 12]. Differences in efficacy between modified FOLFIRINOX and GEM-NAB were assessed by HRs with $95 \%$ Cls. Risk ratios (RRs) with $95 \%$ Cls were used to evaluate the outcomes of ORRs. And the outcome of odds ratios (ORs) were used to assess grade $\geq 3$ toxicities. The random-effect model (DerSimonian-Laird method) was used to calculate the pooled HR, RR and $\mathrm{OR}$ in direct and indirect meta-analysis. HRs were calculated by inverse-variance approach. ORs and RRs were calculated by Mantel-Haenszel method. The indirect comparison was based on the assumption of transitivity $[13,14]$. When direct evidence was lacked between intervention $A$ and intervention $B$, we could obtain indirect comparisons via common intervention $C$ which associated intervention A with intervention B[15]. All calculations were conducted using STATA software[16-18], version 13.1 (StataCorp, College Station, TX, USA) with mvmeta, network, metareg, metan packages (http://fmwww.bc.edu/RePEc/bocode/m).

Node-spilt method was used to assess inconsistency[19]. Global or local heterogeneity among studies were evaluated by calculating $p$ value, which was considered significant if $p$ value was less than $0.05[20]$. Loop inconsistency was estimated by calculating IFs with $95 \%$ Cls, which was considered insignificant if $95 \% \mathrm{Cl}$ included 0 . The possible bias of publication was detected by Egger's test[21], which was considered significant if $p$ value was less than 0.05 .

\section{Results}

\section{Study search}

For initial literature search, a total of 4707 related studies were identified from databases that are available. The detailed flow diagram of study selection process is shown in Fig. 1. We excluded 2223 studies due to duplication. Upon title and abstract screening, 212 studies remained. Only 22 studies involving approximately 6351 patients were eligible after full-text screening and were included to conduct a final meta-analysis[22-43].

The detailed description of included studies is presented in Additional file 1. Four kinds of treatment were analyzed in order to compare the efficacy and safety of modified FOLFIRINOX and GEM-NAB. The number of patients with different treatments ranged from 12 to 632 . The baseline characteristics of patients in studies are also listed in Additional file 1. No overlap of population existed in our analysis.

\section{Overall Survival (os)}

The forest plots of network meta-analysis of OS are demonstrated in Figure S1. Heterogeneity was detected and significant inconsistency was not observed in our data. The comparisons for hazards ratios 
of OS between different treatments are shown in Table 1. The therapy of modified FOLFIRINOX had better survival benefit than the therapy of GEM-NAB (HR: $0.89 ; 95 \% \mathrm{Cl}: 0.61-1.29)$, but this difference was not significant. The use of modified FOLFIRINOX (HR: 0.44; 95\% Cl: 0.29-0.65), GEM-NAB (HR: 0.49; 95\% Cl: 0.39-0.61) and FOLFIRINOX (HR: $0.46 ; 95 \% \mathrm{Cl}: 0.37-0.57$ ) exhibited better treatment efficacy than gemcitabine monotherapy, showing improved mortality risks of $66 \%, 57 \%$ and $60 \%$ respectively.

Table 1

Indirect comparison of overall survival.

\section{OS HR (95\% Cl)}

\section{GEM}

$0.49(0.39,0.61)$ * GEM-NAB

$\begin{array}{llll}0.46(0.37,0.57) * & 0.93(0.80,1.08) & \text { FOLFIRINOX } & \\ 0.44(0.29,0.65) * & 0.89(0.61,1.29) & 1.05(0.75,1.47) & \text { mFOLFIRINOX }\end{array}$

Comparisons between treatments were read from left to right, and the estimate (hazard ratio, HR) with $95 \%$ confidence interval for a given comparison was read in the intersection of two treatments. The value of estimates higher than 1 indicated that column-defining treatment had better efficacy. * Denotes p-value < 0.05. GEM: gemcitabine; GEM-NAB: Gemcitabine plus nab-paclitaxel; FOLFIRINOX: the combination of 5-fluorouracil, oxaliplatin, and irinotecan; mFOLFIRINOX: at least one of the drugs was reduced and/or the removal of 5-FU bolus in FOLFIRINOX.

\section{Progression-free Survival (pfs)}

The forest plots of network meta-analysis of PFS are demonstrated in Figure S2. Heterogeneity was not statistically significant in our analysis. Table 2 demonstrates indirect comparisons of hazards ratios of PFS between treatments, which shows similar results with OS. The survival data on PFS showed better efficacy in the treatment of modified FOLFIRINOX (HR: $0.84 ; 95 \% \mathrm{Cl}: 0.60-1.17$ ) compared to GEM-NAB, but there was no significance statistically. Modified FOLFIRINOX (HR: 0.31 ; $95 \%$ Cl: $0.19-0.51$ ), GEM-NAB (HR: 0.37 ; 95\% Cl: $0.25-0.56$ ) and FOLFIRINOX (HR: $0.31 ; 95 \% \mathrm{Cl}: 0.20-0.48)$ had better treatment effect than gemcitabine monotherapy. 
Table 2

Indirect comparison of progression free survival.

\section{PFS HR (95\% Cl)}

GEM
$0.37(0.25,0.56)$ *
GEM-NAB

$0.31(0.20,0.48)$ *

$0.84(0.66,1.07)$

FOLFIRINOX

$0.31(0.19,0.51)$ *

$0.84(0.60,1.17)$

$1.00(0.78,1.29)$

mFOLFIRINOX

Comparisons between treatments were read from left to right, and the estimate (hazard ratio, HR) with $95 \%$ confidence interval for a given comparison was read in the intersection of two treatments. The value of estimates higher than 1 indicated that column-defining treatment had better efficacy. * Denotes p-value < 0.05. GEM: gemcitabine; GEM-NAB: Gemcitabine plus nab-paclitaxel; FOLFIRINOX: the combination of 5-fluorouracil, oxaliplatin, and irinotecan; mFOLFIRINOX: at least one of the drugs was reduced and/or the removal of 5-FU bolus in FOLFIRINOX.

\section{Objective Response Rate (orr)}

The forest plots of network meta-analysis of ORR are demonstrated in Figure S3. Heterogeneity was not statistically significant in our analysis. The comparisons for risk ratios of ORR between different treatments are shown in Table 3, which were different from the results of OS or PFS. A better objective response rate was observed in GEM-NAB (RR: $0.70 ; 95 \% \mathrm{Cl}: 0.51-0.96)$ than modified FOLFIRINOX. And gemcitabine monotherapy also showed less efficacy than other treatments, either.

Table 3

Indirect comparison of objective response rate.

\section{ORR RR $(95 \% \mathrm{Cl})$}

\section{GEM}

$0.21(0.11,0.40)$ *

GEM-NAB

$0.25(0.13,0.49)$ *

$1.22(0.95,1.58)$

FOLFIRINOX

$0.30(0.15,0.60)$ *

$1.43(1.04,1.96)$ *

$0.86(0.64,1.15)$

mFOLFIRINOX

Comparisons between treatments were read from left to right, and the estimate (risk ratio, RR) with $95 \%$ confidence interval for a given comparison was read in the intersection of two treatments. The value of estimates higher than 1 indicated that column-defining treatment had better efficacy. * Denotes p-value < 0.05 . GEM: gemcitabine; GEM-NAB: Gemcitabine plus nab-paclitaxel; FOLFIRINOX: the combination of 5-fluorouracil, oxaliplatin, and irinotecan; mFOLFIRINOX: at least one of the drugs was reduced and/or the removal of 5-FU bolus in FOLFIRINOX.

\section{Toxicity}


We demonstrated direct and indirect toxicity comparisons between different treatments and listed results in Fig. 2. Heterogeneity was not statistically significant in our analysis $(p<0.05)$. Patients treated with GEM-NAB or modified FOLFIRINOX showed similar risk of adverse events. Increasing risk of neutropenia, febrile neutropenia, thrombocytopenia, anorexia, nausea and diarrhea were observed in patients treated with modified FOLFIRINOX. And patients who received GEM-NAB showed a little higher risk of fatigue and anemia. But these results were not statistically significant. A higher risk of neutropenia was observed in modified FOLFIRINOX (OR: 4.48; 95\% Cl: 2.72-7.37), FOLFIRINOX (OR: 5.70; 95\% Cl: 3.69-8.81) and GEM-NAB (OR: 4.39; 95\% Cl: 2.78-6.94) than gemcitabine monotherapy. Gastrointestinal disorders such as nausea (OR: $27.05 ; 95 \% \mathrm{Cl}: 1.13-648.80$ ) and diarrhea (OR: 40.09; 95\% Cl: 1.70-945.53) were more frequently observed in patients treated with FOLFIRINOX than gemcitabine monotherapy.

\section{Publication Bias}

Publication bias of OS data was assessed by Egger's test $(p=0.918)$. No publication bias was found in our analysis. And results were similar for PFS (Egger's, $p=0.167$ ) and ORR (Egger's, $p=0.267$ ). We further evaluated the potential sources of the heterogeneity by calculating $p$ values. The $p$ values were 0.512 , 0.164 and 0.379 for OS, PFS and ORR, respectively, which showed no heterogeneity was found in our study.

\section{Discussion}

Modified FOLFIRINOX and GEM-NAB are the most frequently used first-line treatments for advanced PC. Previous studies have conducted efficacy comparisons between modified FOLFIRINOX and FOLFIRINOX[9] or between GEM-NAB and FOLFIRINOX[44], and the outcomes were similar in these comparisons. However, until now, relative effects of modified FOLFIRINOX and GEM-NAB treatments are unknown as no direct comparisons have been conducted. This study performed a network meta-analysis of four kinds of treatments including modified FOLFIRINOX, FOLFIRINOX, GEM-NAB and gemcitabine monotherapy on survival outcomes and adverse effects. Thus, our analysis is unique of its kind and could provide some guidance to treatment selection in the first-line setting for advanced PC.

Chemotherapy is the cornerstone of advanced PC due to its invasive biological characteristics. It is known that FOLFIRINOX is one of the standard treatments for advanced PC. Oxaliplatin, irinotecan and 5FU were reported to show synergistic antitumor effects in several studies especially in metastatic PCs[45-47]. In a Phase III clinical trial[48], gemcitabine showed an increase in survival of 5.65 months than 4.41 months of 5-FU. Gemcitabine combined with nab-paclitaxel could lengthen the median OS to 8.5 months compared with 6.7 months in the gemcitabine monotherapy group. In 2013, based on the results of the MPACT trial[5], the GEM-NAB combination was approved for advanced PC in the first-line setting. When compared to gemcitabine alone indirectly, the mOS among patients treated with FOLFIRINOX or GEM-NAB were 11.1 months and 8.5 months, mPFS were 6.4 and 5.5 months, and ORRs were $31.6 \%$ and $23 \%$ respectively in ACCORD and MPACT pivotal studies $[5,10]$. Thus, the therapeutic benefits of FOLFIRINOX were better than GEM-NAB in this cohort. However, previous meta-analysis based 
on 16 retrospective studies from Italy reported that the survival outcomes were similar between FOLFIRINOX and GEM-NAB[44], with HR for PFS of 0.88 (95\% CI 0.71-1.1, $p=0.26)$ and HR for OS of $0.99(95 \% \mathrm{Cl} 0.84-1.16, \mathrm{p}=0.9)$. In our meta-analysis, the treatment efficacy was also similar between FOLFIRINOX and GEM-NAB, which was same with previous studies. FOLFOXIRI, a dosage-modified version of FOLFIRINOX, has been widely utilized and has shown satisfying outcomes in metastatic colorectal cancer[49]. An observational study of 137 patients from Italy reported that median OS and PFS were 8.0 months and 12.0 months respectively with metastatic $P C$ treated with modified FOLFIRINOX (FOLFOXIRI)[50]. In view of all related retrospective studies of modified FOLFIRINOX and GEM-NAB, our indirect meta-analysis showed that survival outcomes were similar in two treatments. The results of OS and PFS showed better treatment efficacy in modified FOLFIRINOX group than GEM-NAB group. But this difference was not statistically significant. As for results of ORR, GEM-NAB demonstrated more benefits than modified FOLFIRINOX, and this difference was statistically significant. Therefore, based on data we collected until now, we could not determine which treatment had better survival outcomes.

Treatment-related adverse effects were much severe in patients who received FOLFIRINOX (grade $\geq 3$ : neutropenia $45.7 \%$, fatigue $23.6 \%$, and diarrhea $12.7 \%$ of patients) than that of GEM-NAB (grade $\geq 3$ : neutropenia $38 \%$, fatigue $7 \%$, and diarrhea $6 \%$ of patients) in the PRODIGE4/ACCORD11 trial[5, 10, 51], which is a barrier in the wide employment of FOLFIRINOX. Thus, dose reduction of FOLFIRINOX have been conducted in several groups to reduce FOLFIRINOX-related toxicities. In a UK retrospective research[52], a lower rate of neutropenia was reported after dosage reduction of irinotecan and omission of 5-FU bolus than that in PRODIGE4/ACCORD11 trial. Dosage reduction of irinotecan and 5-FU bolus were also reported to decrease the risk of neutropenia and vomiting in a US phase II trial[53]. A retrospective research from South Korea found that the toxicity of modified FOLFIRINOX was less severe compared to FOLFIRINOX[27]. A previous meta-analysis from China found that modified FOLFIRINOX could reduce toxicity without compromising treatment efficacy compared to standard FOLFIRINOX[9]. However, the benefits from modified FOLFIRINOX were based only on comparisons with standard FOLFIRINOX. And the choice of modified FOLFIRINOX or GEM-NAB has been controversial due to the lack of evidence comparing the toxicity between two treatments. For grade $\geq 3$ adverse events, our analysis showed that the risk was higher in patients treated with modified FOLFIRINOX than GEM-NAB including neutropenia, febrile neutropenia, thrombocytopenia, anorexia, nausea and diarrhea. And lower risk of fatigue and anemia was observed in the modified FOLFIRINOX group than that of the GEM-NAB group. However, our results did not significantly differ. Our analysis showed a similar toxicity profile in advanced pancreatic cancer patients treated with modified FOLFIRINOX compared to GEM-NAB, and provided some guidance to medical participants in treatment selection.

The latest clinical data were utilized in order to conduct indirect comparisons between different drug combinations. But this study has some clear limitations. Firstly, the results of analysis might be overestimated due to bias of small sample size. Further trials were required to specify the estimation of patients benefited. Secondly, our analysis was based on retrospective studies. Thus, patients were not randomly selected or distributed, and factors including patients age, performance status, tumor burden or disease stage were not adequately evaluated which might cause arms unbalanced. Thirdly, the data in 
terms of patients' baseline characteristics and follow up were not complete. The evaluation of PFS or ORR were subjective to a certain extent with obvious bias in which the heterogeneity could not be avoided. Despite several limitations of this network meta-analysis, it is the initial work comparing efficacy and safety indirectly between modified FOLFIRINOX and GEM-NAB among PC patients.

\section{Conclusions}

On the whole, many factors should be considered for the optimal treatment formulation. GEM-NAB was only utilized when the treatment of adjuvant gemcitabine or gemcitabine-based failed as well that the relapse-free survival (RFS) duration was more than 6 months. And the basic health conditions of patients should be evaluated as GEM-NAB was less toxic compared to triplet therapy. Furthermore, patients should be notified with the costs of supportive care such as apply of anti-emetics or pegfilgrastim[54]. Costs are not only based on chemotherapy drug itself but also on supportive treatment determined by severity and frequency of adverse effects. Previous study found that FOLFIRINOX therapy was more expensive than GEM-NAB[55]. However, comparison of treatment patterns and economic outcomes between modified FOLFIRINOX and GEM-NAB were still unknown. Still, the use of both modified FOLFIRINOX and GEM-NAB as first-line therapy in patients with advanced PC should be promising in the future.

\section{List Of Abbreviations}

GEM: gemcitabine; GEM-NAB: gemcitabine plus nab-paclitaxel; mFOLFIRINOX: modified FOLFIRINOX; PC: pancreatic cancer; OS: overall survival; PFS: progression-free survival; ORR: objective response rate; 5-FU: 5-fluorouracil; CR: complete response; PR: partial response; SD: stable disease; PD: progressive disease; HRs: hazard ratios; RRs: risk ratios; ORs: odds ratios; Cls: confidence intervals.

\section{Declarations}

\section{Ethics approval and consent to participate}

Ethical approval was not required for this study because only previously published data from peerreviewed publications were used. These data do not contain any information that could identify subjects.

\section{Consent for publication}

Not applicable.

\section{Availability of data and materials}

All data generated or analyzed during this study are included in this published article and referenced articles are listed in the References section.

Competing interests 
The authors declare that they have no competing interests.

\section{Funding}

This work was supported by the National Natural Science Foundation of China (grant 81874061), Chinese Society of Clinical Oncology-Shiyao Cancer Research Fund (grant Y-sy2019-009), Hubei Provincial Natural Science Foundation Guiding Project (2018CFC846) and the 7th Wuhan Young and Middle-aged Backbone Talent of Medical Training Project 2019 (2019 No. 87).

\section{Authors' contributions}

JYC, ZYL and TZ obtained funding for the study and conceptualized and designed the study. JYC, QLH, HHW, LZ, DJZ, and DDY collected the data. JYC, QLH, ZYL and TZ screened and extracted the data. DJZ, GLP and HHW conducted data analysis. JYC, DDY, and HHW reviewed the data analysis. JYC drafted the article. ZYL and TZ critically revised the article. All authors have read and approved the final article.

\section{Acknowledgements}

Not applicable

\section{References}

1. Siegel RL, Miller KD, Jemal A: Cancer statistics, 2020. CA Cancer J Clin 2020, 70(1):7-30.

2. Ducreux M, Cuhna AS, Caramella C, Hollebecque A, Burtin P, Goere D, Seufferlein T, Haustermans K, Van Laethem JL, Conroy $T$ et al: Cancer of the pancreas: ESMO Clinical Practice Guidelines for diagnosis, treatment and follow-up. Ann Oncol 2015, 26 Suppl 5:v56-68.

3. Sohal DPS, Kennedy EB, Khorana A, Copur MS, Crane CH, Garrido-Laguna I, Krishnamurthi S, Moravek C, O'Reilly EM, Philip PA et al: Metastatic Pancreatic Cancer: ASCO Clinical Practice Guideline Update. J Clin Oncol 2018, 36(24):2545-2556.

4. Moore MJ, Goldstein D, Hamm J, Figer A, Hecht JR, Gallinger S, Au HJ, Murawa P, Walde D, Wolff RA et al: Erlotinib plus gemcitabine compared with gemcitabine alone in patients with advanced pancreatic cancer: a phase III trial of the National Cancer Institute of Canada Clinical Trials Group. $J$ Clin Oncol 2007, 25(15):1960-1966.

5. Von Hoff DD, Ervin T, Arena FP, Chiorean EG, Infante J, Moore M, Seay T, Tjulandin SA, Ma WW, Saleh $\mathrm{MN}$ et al: Increased survival in pancreatic cancer with nab-paclitaxel plus gemcitabine. N Eng/ J Med 2013, 369(18):1691-1703.

6. Kang J, Hwang I, Yoo C, Kim KP, Jeong JH, Chang HM, Lee SS, Park DH, Song TJ, Seo DW et al: Nabpaclitaxel plus gemcitabine versus FOLFIRINOX as the first-line chemotherapy for patients with metastatic pancreatic cancer: retrospective analysis. Invest New Drugs 2018, 36(4):732-741.

7. Peixoto RD, Ho M, Renouf DJ, Lim HJ, Gill S, Ruan JY, Cheung WY: Eligibility of Metastatic Pancreatic Cancer Patients for First-Line Palliative Intent nab-Paclitaxel Plus Gemcitabine Versus FOLFIRINOX. 
Am J Clin Oncol 2017, 40(5):507-511.

8. Kang H, Jo JH, Lee HS, Chung MJ, Bang S, Park SW, Song SY, Park JY: Comparison of efficacy and safety between standard-dose and modified-dose FOLFIRINOX as a first-line treatment of pancreatic cancer. World J Gastrointest Oncol 2018, 10(11):421-430.

9. Tong H, Fan Z, Liu B, Lu T: The benefits of modified FOLFIRINOX for advanced pancreatic cancer and its induced adverse events: a systematic review and meta-analysis. Sci Rep 2018, 8(1):8666.

10. Conroy T, Desseigne F, Ychou M, Bouche O, Guimbaud R, Becouarn Y, Adenis A, Raoul JL, GourgouBourgade $\mathrm{S}$, de la Fouchardiere $\mathrm{C}$ et al: FOLFIRINOX versus gemcitabine for metastatic pancreatic cancer. N Engl J Med 2011, 364(19):1817-1825.

11. Kiefer C, Sturtz S, Bender R: Indirect Comparisons and Network Meta-Analyses. Dtsch Arzteb/ Int 2015, 112(47):803-808.

12. Kim H, Gurrin L, Ademi Z, Liew D: Overview of methods for comparing the efficacies of drugs in the absence of head-to-head clinical trial data. Br J Clin Pharmacol 2014, 77(1):116-121.

13. Donegan S, Williamson P, D'Alessandro U, Tudur Smith C: Assessing key assumptions of network meta-analysis: a review of methods. Res Synth Methods 2013, 4(4):291-323.

14. Salanti G: Indirect and mixed-treatment comparison, network, or multiple-treatments meta-analysis: many names, many benefits, many concerns for the next generation evidence synthesis tool. Res Synth Methods 2012, 3(2):80-97.

15. White IR, Barrett JK, Jackson D, Higgins JP: Consistency and inconsistency in network meta-analysis: model estimation using multivariate meta-regression. Res Synth Methods 2012, 3(2):111-125.

16. Chaimani A, Higgins JP, Mavridis D, Spyridonos P, Salanti G: Graphical tools for network metaanalysis in STATA. PLoS One 2013, 8(10):e76654.

17. White I: MVMETA: Stata module to perform multivariate random-effects meta-analysis. 2018.

18. White IR: Multivariate random-effects meta-regression: updates to mvmeta. The Stata Journa/2011, 11(2):255-270.

19. Dias S, Welton NJ, Caldwell DM, Ades AE: Checking consistency in mixed treatment comparison meta-analysis. Stat Med 2010, 29(7-8):932-944.

20. Higgins JP, Thomas J, Chandler J, Cumpston M, Li T, Page MJ, Welch VA: Cochrane handbook for systematic reviews of interventions: John Wiley \& Sons; 2019.

21. Egger M, Davey Smith G, Schneider M, Minder C: Bias in meta-analysis detected by a simple, graphical test. BMJ 1997, 315(7109):629-634.

22. Barrera I, Ranger J, Roofigari N, Dalfen R, Batist G, Kavan P: Treatment sequencing in MPC, insights from a $3^{\circ}$ care center. Journal of Clinical Oncology 2019, 37.

23. Chan KKW, Guo H, Cheng S, Beca JM, Redmond-Misner R, Isaranuwatchai W, Qiao L, Earle C, Berry SR, Biagi JJ et al: Real-world outcomes of FOLFIRINOX vs gemcitabine and nab-paclitaxel in advanced pancreatic cancer: A population-based propensity score-weighted analysis. Cancer Med 2020, 9(1):160-169. 
24. Cho I, Kang H, Jo J, Lee H, Chung M, Park J, Park S, Song S, Park M, An C et al: FOLFIRINOX versus gemcitabine plus nab-paclitaxel for treatment of metastatic pancreatic cancer: A single-center cohort study. Annals of Oncology 2018, 29:v45.

25. de Jesus VHF, Camandaroba MPG, Donadio MDS, Cabral A, Muniz TP, de Moura Leite L, Sant'Ana LF: Retrospective comparison of the efficacy and the toxicity of standard and modified FOLFIRINOX regimens in patients with metastatic pancreatic adenocarcinoma. J Gastrointest Oncol 2018, 9(4):694-707.

26. Javed MA, Beyer G, Le N, Vinci A, Wong H, Palmer D, Morgan RD, Lamarca A, Hubner RA, Valle JW et al: Impact of intensified chemotherapy in metastatic pancreatic ductal adenocarcinoma (PDAC) in clinical routine in Europe. Pancreatology 2019, 19(1):97-104.

27. Kang H, Jo JH, Lee HS, Chung MJ, Bang S, Park SW, Song SY, Park JY: Comparison of efficacy and safety between standard-dose and modified-dose FOLFIRINOX as a first-line treatment of pancreatic cancer. World Journal of Gastrointestinal Oncology 2018, 10(11):421-430.

28. Kang J, Hwang I, Yoo C, Kim K-p, Jeong JH, Chang H-M, Lee SS, Park DH, Song TJ, Seo DW et al: Nabpaclitaxel plus gemcitabine versus FOLFIRINOX as the first-line chemotherapy for patients with metastatic pancreatic cancer: retrospective analysis. Investigational New Drugs 2018, 36(4):732-741.

29. Kasi A, Middinti A, Cao A, Vekaria P, Patel D, Subramaniam D, Chen GJ, Saeed AM, Paluri RK: FOLFIRINOX versus gemcitabine nab-paclitaxel for advanced pancreatic cancer: KU Cancer Center experience. Journal of Clinical Oncology 2017, 35.

30. Kim S, Signorovitch JE, Yang H, Patterson-Lomba O, Xiang CQ, Ung B, Parisi M, Marshall JL: Comparative Effectiveness of nab-Paclitaxel Plus Gemcitabine vs FOLFIRINOX in Metastatic Pancreatic Cancer: A Retrospective Nationwide Chart Review in the United States. Adv Ther 2018, 35(10):1564-1577.

31. Latenstein AEJ, Mackay TM, Creemers GJ, van Eijck CHJ, de Groot JWB, Haj Mohammad N, Homs MYV, van Laarhoven HWM, Molenaar IQ, Ten Tije BJ et al: Implementation of contemporary chemotherapy for patients with metastatic pancreatic ductal adenocarcinoma: a population-based analysis. Acta Oncol 2020:1-8.

32. Lee J-C, Shin DW, Yang SY, Kim MJ, Kim J, Woo SM, Lee WJ, Choi KS, Jung JH, Kang J et al: Comparison of FOLFIRINOX and gemcitabine with nab-paclitaxel in metastatic pancreatic cancer: Using Korean Pancreatic Cancer (K-PaC) Registry. Journal of Clinical Oncology 2019, 37(15).

33. Longo Muñoz F, Castillo Trujillo O, Serrano Domingo J, Martín Huertas R, Corral de la Fuente E, San Juan A, Portela J, Cano J, Reguera Puertas P, Rodriguez Garrote M et al: Folfirinox versus nabpaclitaxel plus gemcitabine in the first-line chemotherapy for patients with advanced pancreatic ductal adenocarcinoma: A multivariate analysis of prognostic factors in a national cohort (Comunica-TTD working group). Annals of Oncology 2019, 30:aa92-aa93.

34. Muranaka T, Kuwatani M, Komatsu Y, Sawada K, Nakatsumi H, Kawamoto Y, Yuki S, Kubota Y, Kubo K, Kawahata S et al: Comparison of efficacy and toxicity of FOLFIRINOX and gemcitabine with nab- 
paclitaxel in unresectable pancreatic cancer. Journal of Gastrointestinal Oncology 2017, 8(3):566571.

35. Nakazawa J, Otsuka T, Shimokawa M, Koga F, Ueda Y, Otsu S, Arima S, Fukahori M, Makiyama A, Taguchi $\mathrm{H}$ et al: A multicenter retrospective study of gemcitabine plus nabpaclitaxel or FOLFIRINOX in metastatic pancreatic cancer: NAPOLEON study. Annals of Oncology 2019, 30:aa17-aa18.

36. Ohba A, Ueno H, Sakamoto Y, Kondo S, Morizane C, Okusaka T: Retrospective comparison of modified FOLFIRINOX with full-dose FOLFIRINOX for advanced pancreatic cancer: A Japanese cancer center experience. Journal of Clinical Oncology 2018, 36(4).

37. Papneja N, Zaidi A, Chalchal H, Moser M, Tan K, Olson C, Haider K, Shaw J, Ahmed S: Comparisons of Outcomes of Real-World Patients With Advanced Pancreatic Cancer Treated With FOLFIRINOX Versus Gemcitabine and Nab-Paclitaxel A Population-Based Cohort Study. Pancreas 2019, 48(7):920-926.

38. Tahara J, Shimizu K, Otsuka N, Akao J, Takayama Y, Tokushige K: Gemcitabine plus nab-paclitaxel vs. FOLFIRINOX for patients with advanced pancreatic cancer. Cancer Chemother Pharmacol 2018, 82(2):245-250.

39. Terashima T, Yamashita T, Sakai A, Ohta H, Hinoue Y, Toya D, Kawai H, Yonejima M, Urabe T, Noda Y et al: Treatment patterns and outcomes of unresectable pancreatic cancer patients in real-life practice: a region-wide analysis. Japanese Journal of Clinical Oncology 2018, 48(11):966-973.

40. Vienot A, Chevalier H, Bolognini C, Gherga E, Klajer E, Meurisse A, Jary M, Kim S, d'Engremont C, Nguyen $T$ et al: FOLFOXIRI vs FOLFIRINOX as first-line chemotherapy in patients with advanced pancreatic cancer: A population-based cohort study. World journal of gastrointestinal oncology 2020, 12(3):332-346.

41. Wang Y, Camateros P, Cheung WY: A Real-World Comparison of FOLFIRINOX, Gemcitabine Plus nabPaclitaxel, and Gemcitabine in Advanced Pancreatic Cancers. J Gastrointest Cancer 2019, 50(1):6268.

42. Watanabe K, Hashimoto Y, Umemoto K, Takahashi H, Sasaki M, Imaoka H, Ohno I, Mitsunaga S, Ikeda M: Clinical outcome of modified FOLFIRINOX versus gemcitabine plus nab-paclitaxel as first line chemotherapy in metastatic pancreatic cancer. Journal of Clinical Oncology 2017, 35(4).

43. Williet N, Saint A, Pointet AL, Tougeron D, Pernot S, Pozet A, Bechade D, Trouilloud I, Lourenco N, Hautefeuille $V$ et al: Folfirinox versus gemcitabine/nab-paclitaxel as first-line therapy in patients with metastatic pancreatic cancer: a comparative propensity score study. Therap Adv Gastroenterol 2019, 12:1756284819878660.

44. Pusceddu S, Ghidini M, Torchio M, Corti F, Tomasello G, Niger M, Prinzi N, Nichetti F, Coinu A, Di Bartolomeo $\mathrm{M}$ et al: Comparative Effectiveness of Gemcitabine plus Nab-Paclitaxel and FOLFIRINOX in the First-Line Setting of Metastatic Pancreatic Cancer: A Systematic Review and Meta-Analysis. Cancers (Basel) 2019, 11(4).

45. Ducreux M, Mitry E, Ould-Kaci M, Boige V, Seitz JF, Bugat R, Breau JL, Bouche O, Etienne PL, Tigaud $\mathrm{JM}$ et al: Randomized phase II study evaluating oxaliplatin alone, oxaliplatin combined with 
infusional 5-FU, and infusional 5-FU alone in advanced pancreatic carcinoma patients. Ann Oncol 2004, 15(3):467-473.

46. Conroy T, Paillot B, Francois E, Bugat R, Jacob JH, Stein U, Nasca S, Metges JP, Rixe O, Michel P et al: Irinotecan plus oxaliplatin and leucovorin-modulated fluorouracil in advanced pancreatic cancer-a Groupe Tumeurs Digestives of the Federation Nationale des Centres de Lutte Contre le Cancer study. J Clin Oncol 2005, 23(6):1228-1236.

47. Gourgou-Bourgade S, Bascoul-Mollevi C, Desseigne F, Ychou M, Bouche O, Guimbaud R, Becouarn Y, Adenis A, Raoul JL, Boige $V$ et al: Impact of FOLFIRINOX compared with gemcitabine on quality of life in patients with metastatic pancreatic cancer: results from the PRODIGE 4/ACCORD 11 randomized trial. J Clin Oncol 2013, 31(1):23-29.

48. Burris HA, 3rd, Moore MJ, Andersen J, Green MR, Rothenberg ML, Modiano MR, Cripps MC, Portenoy RK, Storniolo AM, Tarassoff $P$ et al: Improvements in survival and clinical benefit with gemcitabine as first-line therapy for patients with advanced pancreas cancer: a randomized trial. $J$ Clin Oncol 1997, 15(6):2403-2413.

49. Souglakos J, Androulakis N, Syrigos K, Polyzos A, Ziras N, Athanasiadis A, Kakolyris S, Tsousis S, Kouroussis $C$, Vamvakas $L$ et al: FOLFOXIRI (folinic acid, 5-fluorouracil, oxaliplatin and irinotecan) vs FOLFIRI (folinic acid, 5-fluorouracil and irinotecan) as first-line treatment in metastatic colorectal cancer (MCC): a multicentre randomised phase III trial from the Hellenic Oncology Research Group (HORG). Br J Cancer 2006, 94(6):798-805.

50. Vivaldi C, Caparello C, Musettini G, Pasquini G, Catanese S, Fornaro L, Lencioni M, Falcone A, Vasile E: First-line treatment with FOLFOXIRI for advanced pancreatic cancer in clinical practice: Patients' outcome and analysis of prognostic factors. Int J Cancer 2016, 139(4):938-945.

51. Gresham GK, Wells GA, Gill S, Cameron C, Jonker DJ: Chemotherapy regimens for advanced pancreatic cancer: a systematic review and network meta-analysis. BMC Cancer 2014, 14:471.

52. Ghorani E, Wong HH, Hewitt C, Calder J, Corrie P, Basu B: Safety and Efficacy of Modified FOLFIRINOX for Advanced Pancreatic Adenocarcinoma: A UK Single-Centre Experience. Oncology 2015, 89(5):281-287.

53. Stein SM, James ES, Deng Y, Cong X, Kortmansky JS, Li J, Staugaard C, Indukala D, Boustani AM, Patel $\mathrm{V}$ et al: Final analysis of a phase II study of modified FOLFIRINOX in locally advanced and metastatic pancreatic cancer. Br J Cancer 2016, 114(7):737-743.

54. McBride A, Bonafede M, Cai Q, Princic N, Tran O, Pelletier C, Parisi M, Patel M: Comparison of treatment patterns and economic outcomes among metastatic pancreatic cancer patients initiated on nab-paclitaxel plus gemcitabine versus FOLFIRINOX. Expert Rev Clin Pharmacol 2017, 10(10):1153-1160.

55. Kim GP, Parisi MF, Patel MB, Pelletier CL, Belk KW: Comparison of treatment patterns, resource utilization, and cost of care in patients with metastatic pancreatic cancer treated with first-line nabpaclitaxel plus gemcitabine or FOLFIRINOX. Expert Rev Clin Pharmacol 2017, 10(5):559-565. 


\section{Supplementary Materials}

Additional file 1: Characteristics of included studies.

Additional file 2: Detailed search strategy.

Additional file 3: PRISMA NMA checklist of items to include when reporting a systematic review involving a network meta-analysis

\section{Figures}



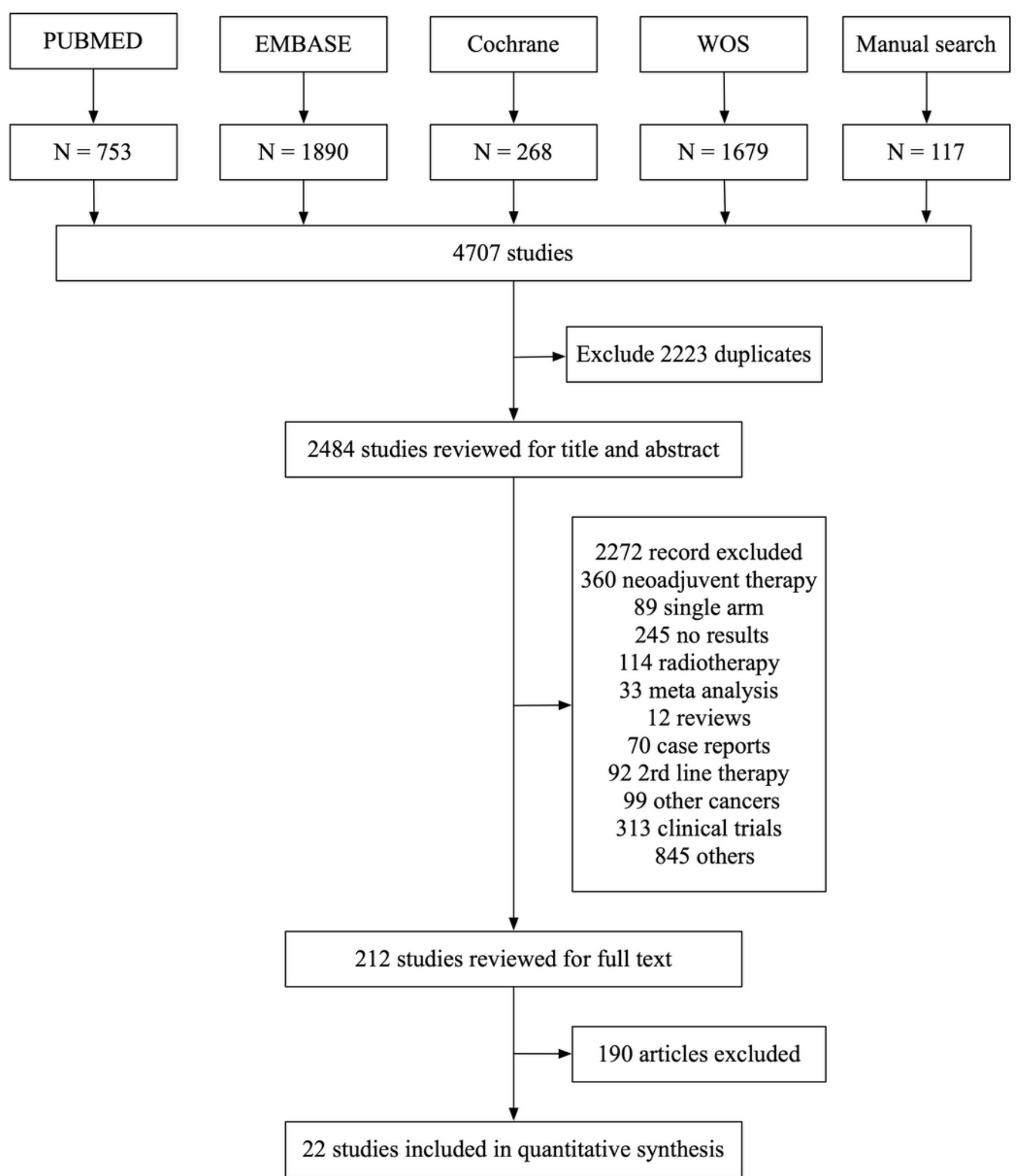

Figure 1

Flow chart for study search (PRISMA diagram). 


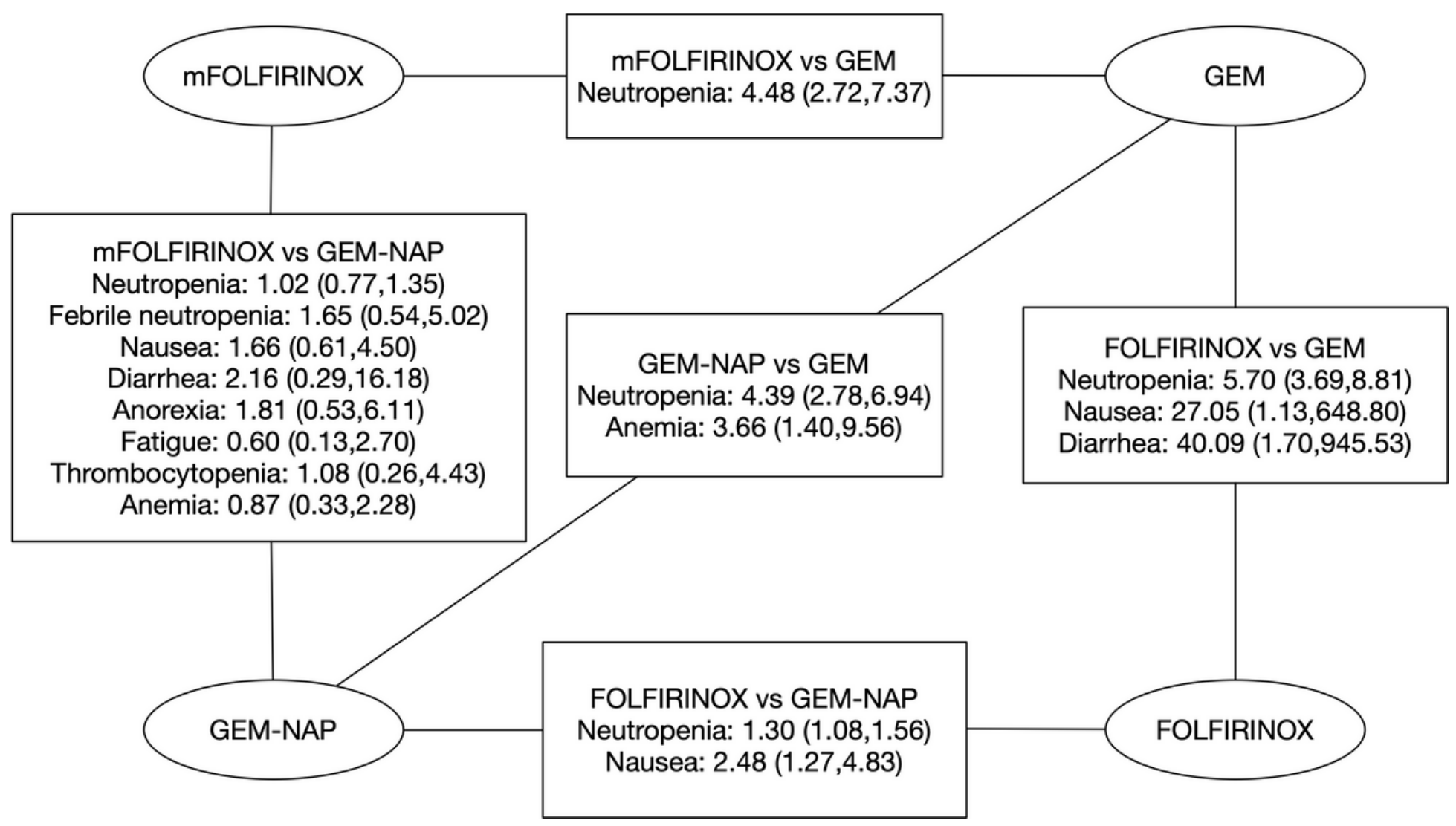

Figure 2

Indirect comparisons of toxicities: modified FOLFIRINOX vs. GEM-NAB. Data presented as odds ratio (OR) with $95 \%$ confidence interval $(\mathrm{Cl})$; the $95 \%$ confidence interval that did not contain the value of 1 represents as statistical significance. Statistically significant comparisons of toxicities between other treatments were also demonstrated.

\section{Supplementary Files}

This is a list of supplementary files associated with this preprint. Click to download.

- FigureS1.tiff

- Figures2.tiff

- FigureS3.tiff

- Additionalfile1.docx

- Additionalfile2.docx

- Additionalfile3PRISMAchecklist.docx 